principle an integral over a Cartan locus is varied by slipping the locus over the cylinder, points going along trajectories, while in the Hamilton principle the same expression is integrated along an arc of a trajectory and variation takes place by keeping the end points fixed but slipping the intervening path over the surface of a cylinder, points going over arcs of Cartan loci.

Finally, two points may be noted.

(1) From the form of (23) Whittaker's remarks which were cited follow as a corollary.

(2) Since (23) does not reduce to (16) as a special case, there are for the restricted conditions to which the original paper applies two different characterizing Hamilton extremal integrals.

The University of Alberta

\title{
NOTE ON THE CANONICAL FORM OF THE PARAMETRIC EQUATIONS OF A SPACE CURVE BELONGING TO A NON- SPECIAL LINEAR LINE COMPLEX
}

\author{
BY C. R. WYLIE, JR.
}

In a recent paper, ${ }^{*}$ the author, by means of a projection from hyper-space, obtained the following equations for a general curve belonging to a linear complex,

$$
\mathrm{A}: \quad x_{1}=-t, \quad x_{2}=f-\frac{1}{2} t f^{\prime}, \quad x_{3}=-f^{\prime}, \quad x_{4}=1 \text {. }
$$

It is the purpose of this note to call attention to a more symmetric form of these equations.

Let $x_{i}=f_{i}(s)$ be the equation of a general space curve, and $P_{13}=P_{42}$ be the equation of a general linear complex. If the curve belongs to the complex

$f_{1} f_{3}^{\prime}-f_{3} f_{1}^{\prime}=f_{4} f_{2}^{\prime}-f_{2} f_{4}^{\prime}$, or $\frac{f_{1}^{2}\left(f_{1} f_{3}^{\prime}-f_{3} f_{1}^{\prime}\right)}{f_{4}^{2} \cdot f_{1}^{2}}=\frac{\left(f_{4} f_{2}^{\prime}-f_{2} f_{4}^{\prime}\right)}{f_{4}^{2}}$

* C. R. Wylie, Jr., Space curves belonging to a non-special linear line complex, American Journal of Mathematics, vol. 57 (1935), pp. 937-942. 
this last can be written in the form

$$
\left(\frac{f}{f_{4}}\right)^{2} \cdot u^{\prime}=v^{\prime},
$$

where $u=f_{3} / f_{1}$ and $v=f_{2} / f_{4}$. From these we have at once

B. 1: $f_{1}=\left(\frac{v^{\prime}}{u^{\prime}}\right)^{1 / 2} \cdot f_{4}, f_{2}=v \cdot f_{4}, f_{3}=u\left(\frac{v^{\prime}}{u^{\prime}}\right)^{1 / 2} \cdot f_{4}, f_{4}=f_{4}$,

B. 2: $x_{1}=\left(v^{\prime}\right)^{1 / 2}, \quad x_{2}=v\left(u^{\prime}\right)^{1 / 2}, \quad x_{3}=u\left(v^{\prime}\right)^{1 / 2}, \quad x_{4}=\left(u^{\prime}\right)^{1 / 2}$.

To show the equivalence of $\mathrm{A}$ and B.2, make the substitutions
C.1: $\quad\left(\frac{v^{\prime}}{u^{\prime}}\right)^{1 / 2}=t$,
C. 2: $\quad \frac{d f}{d t}=u\left(\frac{v^{\prime}}{u^{\prime}}\right)^{1 / 2}=u t$.

From C.1 and C.2 we obtain after an easy integration by parts

$$
v=t \cdot \frac{d f}{d t}-2 f
$$

Using these expressions in B.1 we find

$\mathrm{D}: \quad x_{1}=t, \quad x_{2}=t f^{\prime}-2 f, \quad x_{3}=f^{\prime}, \quad x_{4}=1$,

where now the primes indicate differentiation with respect to $t$ instead of $s$. The slight difference between $\mathrm{D}$ and $\mathrm{A}$ is due to the fact that in the paper mentioned above, the equation of the complex to which the curve belongs was taken to be $P_{13}+2 P_{42}=0$.

Ohio State University 